University of Nebraska - Lincoln

DigitalCommons@University of Nebraska - Lincoln

10-1989

\title{
Angular and Energy Distributions of Electrons from 7.5- 150-keV Proton Collisions with Oxygen and Carbon Dioxide
}

\author{
Wen-Qin Cheng \\ University of Nebraska - Lincoln \\ M. Eugene Rudd \\ University of Nebraska - Lincoln, erudd@unl.edu \\ Ying-Yuan Hsu \\ University of Nebraska - Lincoln
}

Follow this and additional works at: https://digitalcommons.unl.edu/physicsrudd

Part of the Physics Commons

Cheng, Wen-Qin; Rudd, M. Eugene; and Hsu, Ying-Yuan, "Angular and Energy Distributions of Electrons from 7.5- 150-keV Proton Collisions with Oxygen and Carbon Dioxide" (1989). M. Eugene Rudd Publications. 4.

https://digitalcommons.unl.edu/physicsrudd/4

This Article is brought to you for free and open access by the Research Papers in Physics and Astronomy at DigitalCommons@University of Nebraska - Lincoln. It has been accepted for inclusion in M. Eugene Rudd Publications by an authorized administrator of DigitalCommons@University of Nebraska - Lincoln. 


\title{
Angular and energy distributions of electrons from $7.5-150-k e V$ proton collisions with oxygen and carbon dioxide
}

\author{
Wen-Qin Cheng, ${ }^{*}$ M. E. Rudd, and Ying-Yuan Hsu \\ Department of Physics and Astronomy, University of Nebraska-Lincoln, Lincoln, Nebraska 68588-0111
}

(Received 5 April 1989)

\begin{abstract}
Cross sections for the ejection of electrons, differential in the angle and energy of emission, were measured for proton collisions with two molecular gases, oxygen and carbon dioxide, over the primary energy range of 7.5-150 keV and an angular range of $10^{\circ}$ to $160^{\circ}$. The energy distributions, obtained by integration over the angle, were fitted by an analytical model. A discrepancy in the angular distributions compared to those of Gibson and Reid [J. Phys. E 17, 1227 (1984); J. Phys. B 19, 3265 (1986); Radiat. Res. 112, 418 (1987); Australian Atomic Energy Commission Report No. AAEC/E659, 1987 (unpublished)] is discussed. At energies up to 50 or $100 \mathrm{keV}$, the angular distributions were found to be largely independent of the ejected electron energy and very similar for different targets.
\end{abstract}

\section{INTRODUCTION}

The problem of determining the deposition of energy in matter traversed by ions is very old but has not yet received a completely satisfactory solution. Ions lose energy in collisions by a number of different processes, but most of the energy loss from protons, especially at energies above about $50 \mathrm{keV}$, is due to ionization, and at lower energies ionization still remains an important process. Total ionization cross sections for proton impact on most of the simple gases have been measured, and in a recent compilation ${ }^{1}$ recommended values were given over a wide range of energies. However, to construct models of energy deposition, it is necessary to know the differential as well as the total cross sections. Most important are the energy distributions of secondary electrons as given by the singly differential cross sections (SDCS), but in some applications the angular as well as the energy distributions are needed. These are given by the doubly differential cross sections (DDCS) which may be integrated over angle to obtain the SDCS. A second integration yields the total cross section for electron ejection.

DDCS can be calculated using various modifications of the Born approximation. Kuyatt and Jorgensen ${ }^{2}$ carried out the necessary integration for the case of hydrogenic wave functions. By scaling according to the ionization potential, Rudd and Jorgensen ${ }^{3}$ applied those results to helium. A more elaborate calculation using the distorted-wave Born approximation was made by Madison. ${ }^{4}$ Senger ${ }^{5}$ has adapted the plane-wave Born approximation to the treatment of molecules as targets. This was done by summing the partial DDCS for the atomic components of the molecular orbitals. Corrections were made for the effect of the projectile ion on the binding energies and for the post-collision interaction between the projectile and the ejected electron. None of these treatments, however, is useful when the projectile velocity is small compared to the orbital velocity.

Semiempirical methods have been used to obtain ex- pressions for the SDCS. A number of approaches have been suggested which combine the results of Bethe's expansion of the Born approximation with one of the classical binary-encounter approximation (BEA) formulations. Khare and co-workers ${ }^{6,7}$ merged the first term of the Bethe expansion with the Rutherford equation by multiplying each by arbitrary functions and adding. The functions were chosen for best agreement with experiment. They have applied the method to proton collisions with water vapor and with molecular nitrogen. Miller et al. ${ }^{8}$ have combined the Bethe expansion with a more elaborate BEA expression but in a different way in which the second term in the Bethe expansion is replaced by the BEA expression. In both of these models the first Bethe term is obtained from differential optical oscillator strengths obtained from photoionization measurements. A model developed by Inokuti and Dillon ${ }^{9}$ is based entirely on the Bethe theory. The first two Bethe coefficients are expanded in polynomial expressions, each utilizing six adjustable parameters. The fitting parameters are chosen to fit existing data.

These models are all designed for high-energy impact and do not adequately represent cross sections for proton energies below about $100 \mathrm{keV}$ and except for the model of Miller et al., do not reproduce the rapid decrease in the cross section with electron energy above the kinematic cutoff. As the primary energy is lowered, this cutoff comes at lower secondary energies and ultimately the region above the cutoff dominates the entire spectrum.

A semiempirical model has been proposed by Rudd ${ }^{10,11}$ which holds for all primary and secondary energies. In this model, a simple BEA expression was modified to agree asymptotically with the Bethe expression at large impact energies and was further modified in accord with the results of the Fano-Lichten ${ }^{12,13}$ molecular promotion model at secondary energies above the cutoff. Three adjustable parameters suffice to fit the entire secondary energy spectrum at a given impact energy and ten are able to fit the SDCS over the entire range of both primary and 
secondary energies. Since these parameters are primarily determined from experiment, the predictive value of this model is limited. However, it is useful for averaging, interpolating, and extrapolating existing data, and allows a large amount of data to be described in terms of a small number of parameters.

Because no comprehensive a priori methods are available to calculate either SDCS or DDCS for low-energy proton impact, there continues to be a need for experimental data. Proton data for diatomic and triatomic gases are especially scarce. The only available measurements on the two present gases were by Crooks and Rudd, ${ }^{14}$ who measured DDCS for oxygen from 50 to 300 $\mathrm{keV}$ and by Gibson and Reid, ${ }^{15}$ who made measurements on oxygen and carbon dioxide, both at a single energy, 50 $\mathrm{keV}$. The present measurements on these two targets encompasses the range of $7.5-150 \mathrm{keV}$. All of the available data on the two targets are used to obtain parameters for the model.

\section{EXPERIMENTAL RESULTS}

The apparatus and experimental method are the same as that described recently ${ }^{16}$ by the same authors.

\section{A. Singly differential cross sections}

The present data for oxygen are compared to data of Gibson and Reid ${ }^{15}$ at $50 \mathrm{keV}$ and with that of Crooks and Rudd $^{14}$ at $150 \mathrm{keV}$ in Fig. 1. The cross sections are presented as ratios to the Rutherford cross section per electron as suggested by $\mathrm{Kim}$ and Inokuti. ${ }^{17}$ Thus the quantity $Y(E, T)=T E^{2} \sigma(W) / 4 \pi a_{0}^{2} R^{2}$ is plotted where $W$ is the ejected electron energy, $T=m_{e} v_{p}^{2} / 2, m_{e}$ the electron mass, $v_{p}$ the projectile velocity, $a_{0}$ the Bohr radius, and $R$ the Rydberg energy. $E$ is the energy transfer given by $E=W+I_{1}$ where $I_{1}$ is the binding energy of the electrons in the least tightly bound shell. The experimental data sets have each been adjusted by an algorithm ${ }^{11}$ to yield an integrated cross section agreeing with the recommended total cross section. ${ }^{1}$ It is seen that the agreement of the SDCS among the three measurements is very good.

The data have been fitted by the semiempirical model given by Rudd. ${ }^{11}$ The model requires knowledge of the number of electrons in each subshell and their corresponding binding energies, $I$. Data on the binding energies for $\mathrm{O}_{2}$ and $\mathrm{CO}_{2}$ were obtained from Siegbahn et al. ${ }^{18}$ and from Kimura et al. ${ }^{19}$ and are given in Table I. In some cases, subshells with nearly the same binding energies were grouped together. The fitting parameters are given in Table II. These have been determined by making least-squares fits to all available SDCS data and are consistent with recommended values of total cross sections ${ }^{1}$ at all energies. Using these parameters, the model yields SDCS at all combinations of incident proton and ejected electron energies. The results for oxygen are shown in Fig. 1. The contributions to the cross sections from the various subshells are indicated by dashed lines and the total by solid lines. The arrows indicate the points where the secondary electron velocity equals the projectile velocity. The small discrepancy seen at that

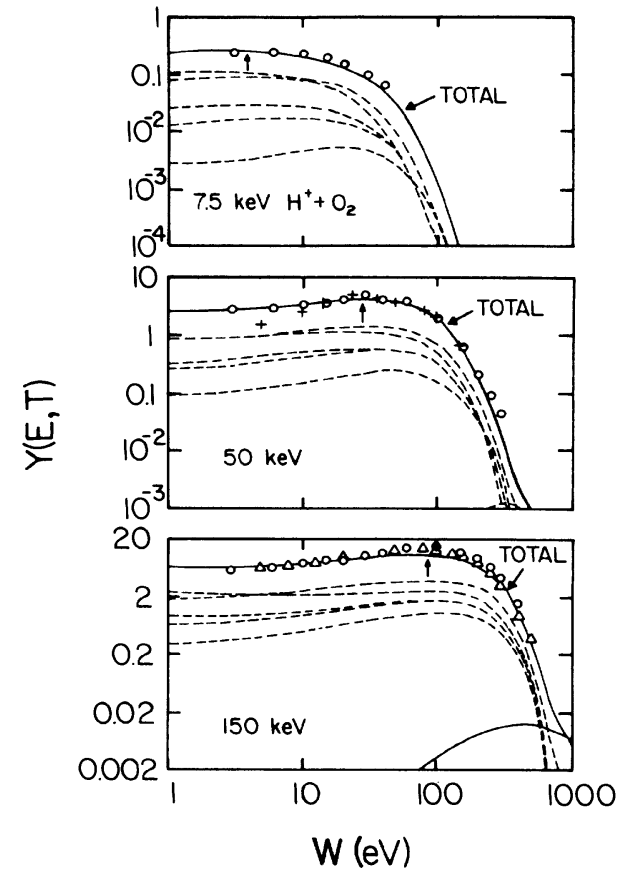

FIG. 1. Energy distributions of electrons, integrated over angle, from 7.5-, 50-, and $150-\mathrm{keV} \mathrm{H}^{+}+\mathrm{O}_{2}$ collisions. $Y(E, T)$ is the ratio of the measured or calculated cross section to the Rutherford cross section. $\bigcirc$, present data; + , data of Gibson and Reid (Ref. 15); $\triangle$, data of Crooks and Rudd (Ref. 14); dashed lines, contributions from various subshells calculated from the model (Ref. 11); solid line, total. The arrows indicate the energies where $v_{e}=v_{p}$.

point in several spectra is due to the mechanism of electron capture to the continuum ${ }^{20}$ which is not included in the model. The SDCS data for $\mathrm{CO}_{2}$ have been treated similarly and are shown in Fig. 2 where they are compared with data of Gibson and Reid and with model calculations. The arrow labeled $A$ indicates the position of the oxygen $K$ Auger peak. Except for these expected discrepancies, the model fits the data very well.

TABLE I. Numbers of electrons and binding energies.

\begin{tabular}{lllc}
\hline \hline Target & Shell & $N$ & $I(\mathrm{eV})$ \\
\hline $\mathrm{O}_{2}$ & $\pi_{g} 2 p$ & 2 & 13.1 \\
& $\pi_{u} 2 p$ & 4 & 17.4 \\
& $\sigma_{g} 2 p$ & 2 & 20.0 \\
& $\sigma_{u} 2 s$ & 2 & 26.6 \\
& $\sigma_{g} 2 s$ & 2 & 40.6 \\
& $\mathrm{O} 1 s$ & 4 & 544 \\
$\mathrm{CO}_{2}$ & $1 \pi_{g}$ & 4 & 13.8 \\
& $1 \pi_{u}$ & 4 & 17.6 \\
& $3 \sigma_{u}$ & 2 & 18.1 \\
& $4 \sigma_{g}$ & 2 & 19.4 \\
& $2 \sigma_{u}$ & 2 & 37.0 \\
& $3 \sigma_{g}$ & 2 & 38.6 \\
& $\mathrm{C} 1 s$ & 2 & 297.5 \\
& $\mathrm{O} 1 s$ & 4 & 541.1 \\
\hline \hline
\end{tabular}


TABLE II. Basic parameters for fitting equation.

\begin{tabular}{cccc}
\hline & $\mathrm{O}_{2}$ & $\mathrm{CO}_{2}$ & Inner shells \\
\hline$A_{1}$ & 1.02 & 1.09 & 1.25 \\
$B_{1}$ & 50.0 & 25.0 & 0.5 \\
$C_{1}$ & 0.4 & 0.75 & 1.0 \\
$D_{1}$ & 0.12 & 0.75 & 1.0 \\
$E_{1}$ & 0.3 & 0.65 & 3.0 \\
$A_{2}$ & 1.0 & 0.78 & 1.1 \\
$B_{2}$ & 5.0 & 3.0 & 1.3 \\
$C_{2}$ & 0.55 & 0.7 & 1.0 \\
$D_{2}$ & 0.0 & 0.85 & 0.0 \\
$\alpha$ & 0.59 & 0.53 & 0.66 \\
\hline \hline
\end{tabular}

\section{B. Doubly differential cross sections}

Since the SDCS are well described by the model, the DDCS may be referenced to them through the quantity $f(\theta)=\sigma(W, \theta) / \sigma(W)$. This quantity is plotted for 20$\mathrm{keV}$ impact energy for $\mathrm{O}_{2}$ and $\mathrm{CO}_{2}$ in Fig. 3 where it is also compared with corresponding data on $\mathrm{N}_{2}$ published earlier. ${ }^{13}$ There are no systematic differences among the three gases when plotted this way. The function $f(\theta)$ is also quite similar for different secondary energies. Figure 4 shows this function for different electron and proton energies for $\mathrm{CO}_{2}$. The differences are generally small and random.

Gibson and Reid ${ }^{15}$ have presented data on $\mathrm{O}_{2}$ and $\mathrm{CO}_{2}$ for $50 \mathrm{keV}$. As Figs. 1 and 2 show, their $\mathrm{O}_{2}$ and $\mathrm{CO}_{2}$ data integrated over all angles agree well with the present SDCS data and with the model calculations. Figures 5 and 6 show a comparison of the angular distribution among the three sets of data which is typical for other energies and targets. While the two sets of data from this laboratory, using different apparatus, agree quite well, there is a pronounced discrepancy with the data of Gibson and Reid. At $10^{\circ}$ their data are higher by a factor of about 1.5 , while at $90^{\circ}$ our data are higher by a factor of about 2.5. These two discrepancies approximately cancel out in the integral over angle so that the SDCS show good agreement. In their paper they attribute the largeangle discrepancy to electrons reflected from target gas molecules and from the chamber walls in our apparatus, but give no explanation for the discrepancy at the small

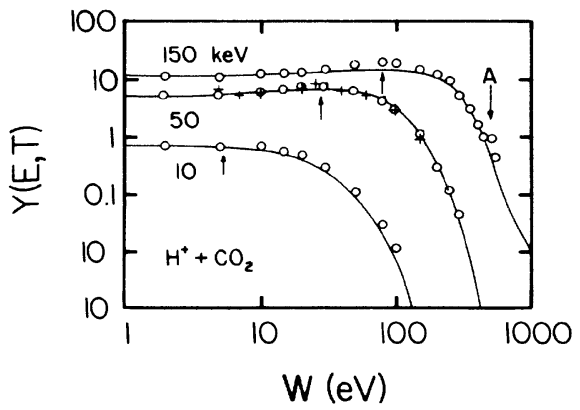

FIG. 2. Energy distributions of electrons, integrated over angle, from 10-, 50-, and $150-\mathrm{keV} \mathrm{H}^{+}+\mathrm{CO}_{2}$ collisions. Legend as in Fig. 1. Position of oxygen Auger peak indicated by $A$.

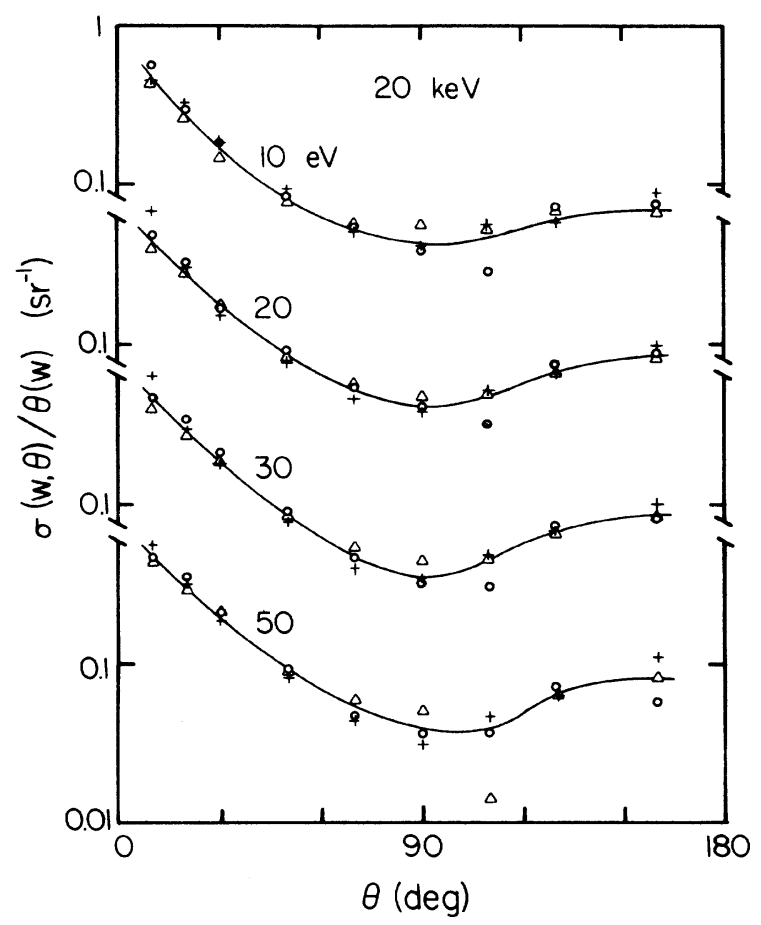

FIG. 3. Angular distributions of electrons of various energies from $20-\mathrm{keV} \mathrm{H}^{+}$collisions with three molecular targets. $\bigcirc$, $\mathrm{CO}_{2} ;+, \mathrm{O}_{2} ; \triangle, \mathrm{N}_{2}$ (Ref. 13). Data are plotted as ratios of the DDCS to the SDCS at each energy.

angles. Their proposed explanation and other possibilities will be examined next.

\section{Sources of error}

The following possible sources of error in the present apparatus have been investigated in some detail: (1) elastic scattering of secondary electrons by the target gas, (2) elastic scattering of secondary electrons by the chamber walls, (3) the production of secondary electrons by protons striking solid surfaces, and (4) the effect of backscattering of protons from the Faraday cup. In each case, plausible or worst-case assumptions were made which allowed an estimate of the error.

\section{Elastic scattering by the target gas}

Secondary electrons are produced all along the beam path in the target gas, a length of $7.5 \mathrm{~cm}$ in our apparatus, but because few electrons are ejected into the backward hemisphere, only the $2.5 \mathrm{~cm}$ up to the collision center is important. If any secondaries enter the space viewed by the detector and scatter in the proper direction while inside that region, they will be detected. This region is small (approximately a $5 \times 5-\mathrm{mm}^{2}$ cylinder), so that only about $10 \%$ of the electrons will enter it and a very small fraction of those will be scattered into the small solid angle of the analyzer entrance slit. The overall fractional error due to this effect is about $10^{-4}$, much too small to be detectable. 


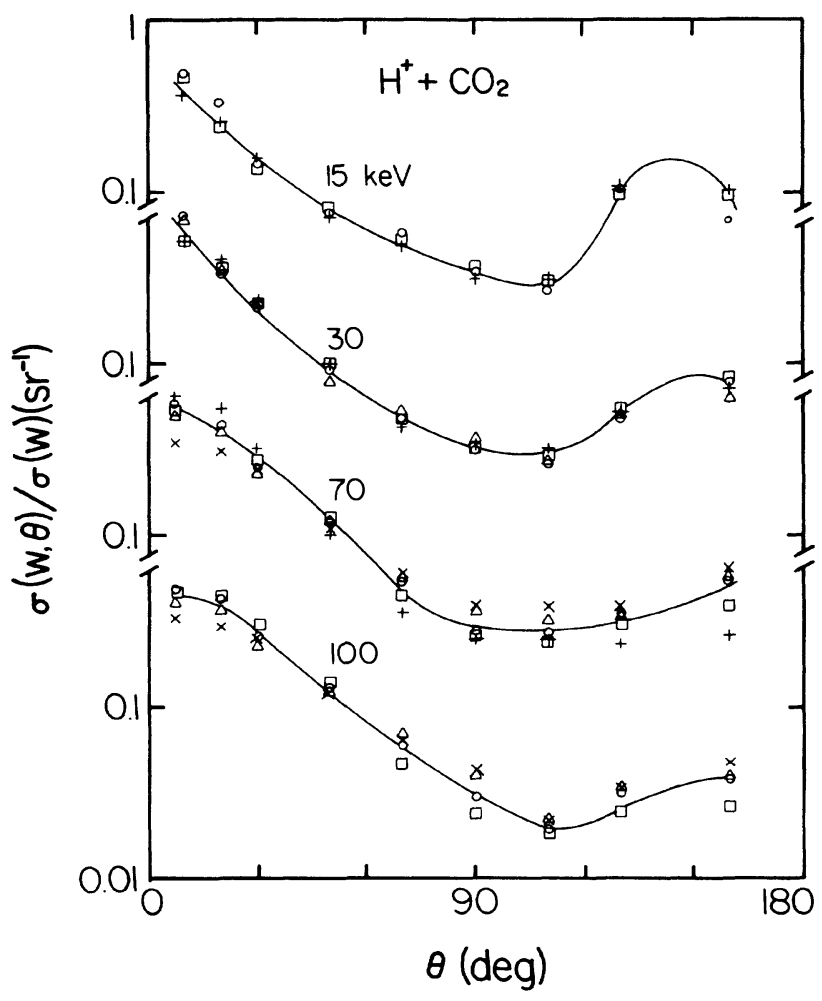

FIG. 4. Comparisons of angular distributions of electrons ejected at various energies from $\mathrm{H}^{+}$collisions with $\mathrm{CO}_{2}$ at 15 , 30,70 , and $100 \mathrm{keV}$. The ratio of the DDCS to the SDCS is plotted for ejected electron energies as follows: $\times, W=T / 4$; $\triangle, W=T / 2 ; \bigcirc, W=T ; \square, W=2 T ;+, W=4 T$, where $T$ is the energy of an electron with the same velocity as the projectile. The lines are only to guide the eye.

\section{Elastic scattering by the chamber walls}

Unfortunately, little information is available on the absorption coefficient $a$ for various surfaces for low-energy electrons. McGowan ${ }^{21}$ has made measurements for several surfaces for 3-50-eV electrons and finds values of $a$ ranging from $0.1-0.6$ at $50 \mathrm{eV}$, the energy for which $a$ was smallest. While none of the surfaces in that investigation was exactly the same as ours, we will take these as representative values in calculating the error. When the absorption coefficient is small, the electrons make many reflections before disappearing so we can assume that they form a swarm moving randomly in all directions. It then becomes a geometric problem to calculate the error. The ratio of the total length of the beam path in the target gas to that viewed by the detector is about 26 . The ratio of the area of wall seen by the detector to the total area inside the chamber is about $10^{-3}$, and the ratio of solid angles subtended by the detection system at the collision center to that at the far wall is 0.5 . Using these and additional data on the angular distribution of the cross sections, we calculate a worst-case error of $0.048 / a$. Using the range of values mentioned above for $a$, we get an error in the range of $8-48 \%$, much too small to explain the factor of 2.5. Furthermore, since $a$ is strongly

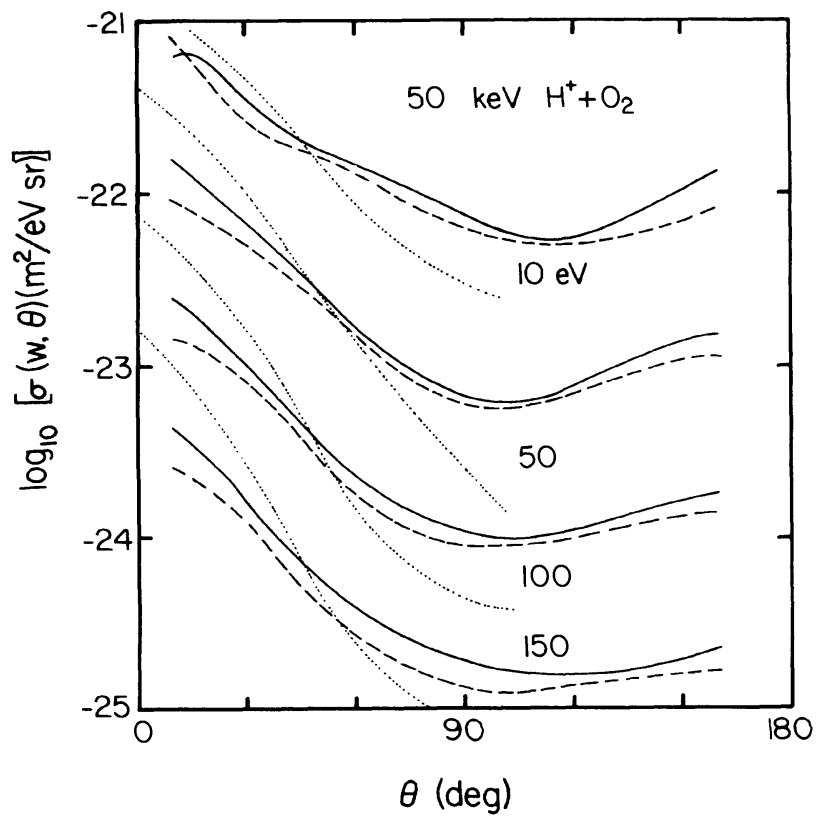

FIG. 5. Angular distributions of electrons from 50-keV $\mathrm{H}^{+}+\mathrm{O}_{2}$ collisions. Solid lines, present data; dotted lines, data of Gibson and Reid (Ref. 15); dashed lines, data of Crooks and Rudd (Ref. 14).

electron-energy dependent, any discrepancy due to this effect should be also. However, as Fig. 5 shows, the discrepancy is nearly the same for all secondary electron energies.

\section{Secondary electrons from protons striking surfaces}

Protons in the present energy range have a secondary electron emission coefficient from surfaces of about 1 , but the energy distribution of secondaries is peaked at 3-4 $\mathrm{eV}$ and falls to a very small value at $25 \mathrm{eV}$. Since the discrepancy to be explained is of approximately the same size for electron energies even up to $150 \mathrm{eV}$, this could not be the source of the discrepancy. In addition, the proton beam was collimated so that protons could only strike the Faraday cup. Since the cup was biased positively at $67.5 \mathrm{~V}$, only a negligibly small fraction of electrons produced there would have sufficient energy to escape and an even smaller fraction would find their way into the analyzer to be detected. The number of protons scattered outside the cup by collisions with target gas molecules is negligibly small at $50 \mathrm{keV}$ and the number scattered from the defining apertures should also be small since they were machined to knife edges to present little area for reflecting beam particles.

\section{Beam particles backscattered from the Faraday cup}

The reflection coefficient for $50-\mathrm{keV}$ protons from copper is about $1 \%$. Since the forward cross section for electron ejection is larger than that in the backward direction, a fraction of the protons moving in the oppo- 


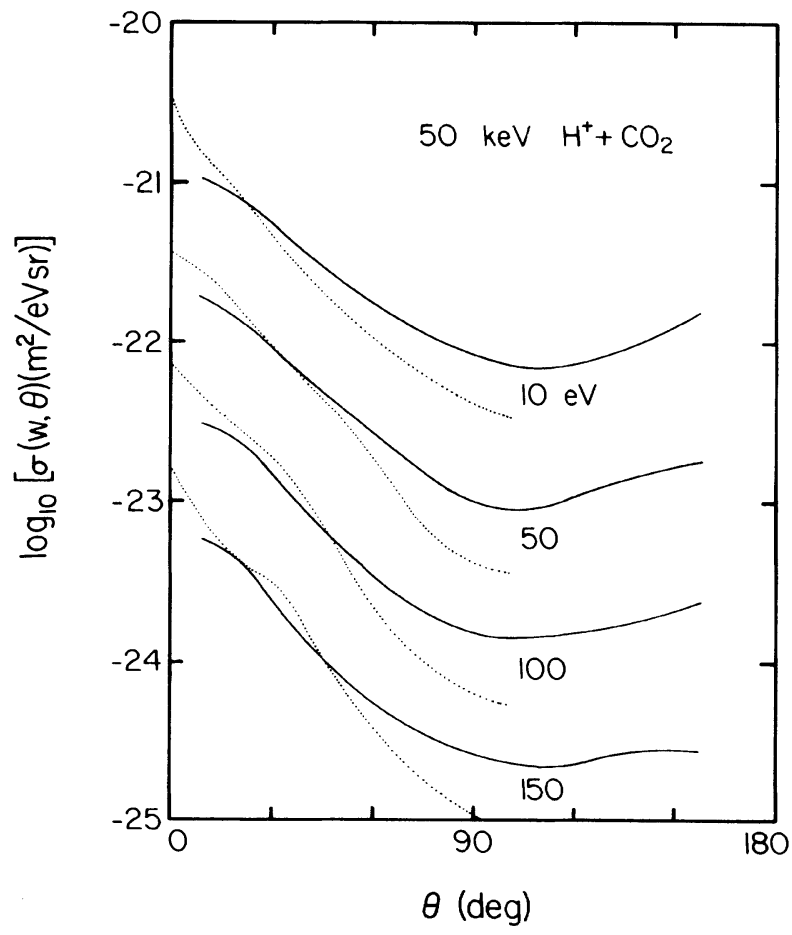

FIG. 6. Angular distributions of electrons from 50-keV $\mathrm{H}^{+}+\mathrm{CO}_{2}$ collisions. Legend as for Fig. 4 .

site direction could have an influence on the measurements at large angles. Because our Faraday cup has a depth-to-radius ratio greater than 10 , any reflected protons must be within an angle of $5.5^{\circ}$ to escape from the cup. Assuming a $\cos ^{2}$ distribution of reflected ions, only about $1.4 \%$ of those reflected will then escape. Using this information and the front-to-back ratio of the electron production cross sections (about 100), the error is about $1.4 \%$. However, because the reflected protons are greatly degraded in energy (they typically have only a few $\mathrm{keV}$ of energy) their effect should be even smaller.

The conclusion is that of these four possible sources of error, only the reflection of electrons from chamber walls could contribute appreciably to the error in the measurements made with the present apparatus. Even this error is much too small to explain the discrepancy at large angles. Furthermore, the error is in the wrong direction to explain the discrepancy at small angles.

Another indication that data from the present apparatus are not subject to such a large error comes from a comparison with other measurements. In the case of argon, four sets of 50-keV DDCS data are available. One set was taken by $\operatorname{Rudd}^{22,23}$ with the same apparatus as the present data. Another was the measurement by Crooks and Rudd ${ }^{14}$ which, although made in the same laboratory, was done with a different apparatus. The third set, by Criswell and Toburen, ${ }^{22,23}$ was made at a different laboratory with an apparatus which used a directed-beam gas target. This measurement, as with that of Gibson and Reid, should therefore be less affected by a possible error due to reflected secondary electrons.
Yet, as Fig. 7 shows, all three of these sets of data agree within $\pm 20 \%$ over the entire angular range, while the measurement by Gibson and Reid shows a discrepancy similar to that described above for oxygen and carbon dioxide.

To further ascertain the extent of a possible error in our cross sections due to elastic scattering of electrons, a system of baffles was installed inside the target cell of the present apparatus. This was made in such a way that the electron detection system looked into a small hole in a cavity which thus formed essentially a blackbody for electrons. The cross sections for electrons ejected at $90^{\circ}$ from $50-\mathrm{keV} \mathrm{H}^{+}+\mathrm{Ar}$ collisions measured with and without the baffles were found to be the same within the $15 \%$ uncertainty over almost the entire energy range of electrons.

All of these lines of evidence seem to indicate that the source of the discrepancy is to be found in the experiment of Gibson and Reid. In their apparatus the proton beam goes directly through a parallel-plate electrostatic analyzer at an angle of $30^{\circ}$ to the plates. Electrons ejected at various angles are deflected to different detectors by the field of the analyzer. While their gas beam is symmetric relative to the various detectors, their proton beam is not. The length of ion beam viewed by their analyzer is approximately $(4 \mathrm{~mm}) / \sin \theta$, where $\theta$ is the angle between the direction of ejection of the electrons being viewed and the proton beam. This gives a length of $18 \mathrm{~mm}$, e.g., at $10^{\circ}$ but only $4 \mathrm{~mm}$ at $90^{\circ}$. If the gas beam were perfectly defined within the $2-\mathrm{mm}$ size that they claim, this would not matter, but gas beams are not always well confined and their claim is based only on a calculation. They describe a procedure for subtracting out the contribution of the background gas but this would not correct for any spread in the gas beam itself.

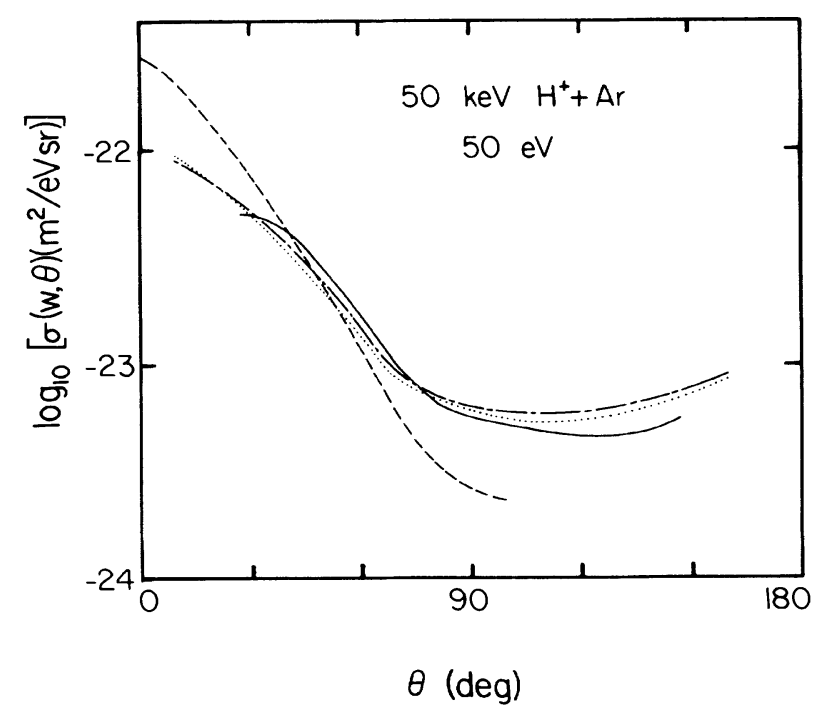

FIG. 7. Comparison of angular distributions of 50-eV electrons from $50-\mathrm{keV} \mathrm{H}^{+}+\mathrm{Ar}$ collisions from four measurements. Solid line, Criswell and Toburen (Refs. 22 and 23); dashed line, Gibson and Reid (Refs. 15); dash-dot line, Crooks and Rudd (Ref. 14); dotted line, Rudd (Refs. 22 and 23). 


\section{CONCLUSIONS}

Data on singly and doubly differential cross sections for electron ejection from $\mathrm{H}^{+}+\mathrm{O}_{2}, \mathrm{CO}_{2}$ collisions have been presented. The SDCS agree well with earlier data from this and other laboratories for the energies where they overlap. The SDCS are described well by a semiempirical analytical model with the sets of parameters given. The angular distributions, described by the ratio of the DDCS to the SDCS at the same secondary energy, show remarkably little variation among the various targets and secondary energies for impact energies up to about 50 or $100 \mathrm{keV}$. Possible sources of error in the experiment were carefully examined to try to resolve the discrepancy in the angular distribution between our data and that of Gibson and Reid. Several possible explanations were ruled out. We suggest that the source of the discrepancy may be the spreading of the target gas beam in the interaction region in their apparatus.

\section{ACKNOWLEDGMENTS}

The authors wish to thank Duane Jaecks for useful discussions and D. K. Gibson for sending tables of data and for helpful correspondence. This paper is based on work supported by National Science Foundation Grants No. PHY-8401328 and No. PHY-8701905.
*Present address: Institute of Physics, Chinese Academy of Sciences, P.O. Box 603, Beijing, China.

${ }^{1}$ M. E. Rudd, Y.-K. Kim, D. H. Madison, and J. W. Gallagher, Rev. Mod. Phys. 57, 965 (1985).

${ }^{2}$ C. E. Kuyatt and T. Jorgensen, Jr., Phys. Rev. 130, 1444 (1963).

${ }^{3}$ M. Eugene Rudd and Theodore Jorgensen, Jr., Phys. Rev. 131, 666 (1963).

${ }^{4}$ M. E. Rudd and D. H. Madison, Phys. Rev. A 14, 128 (1976).

${ }^{5}$ B. Senger, Z. Phys. D 9, 79 (1988).

${ }^{6}$ S. P. Khare and A. Kumar, Physica 100C, 135 (1980).

${ }^{7}$ Y. D. Kaushik, S. P. Khare, and A. Kumar, Physica 106C, 128 (1981).

${ }^{8}$ J. H. Miller, L. H. Toburen, and Steven T. Manson, Phys. Rev. A 27, 1337 (1983).

${ }^{9}$ Mitio Inokuti and Michael A. Dillon, J. Chem. Phys. 87, 6967 (1987).

${ }^{10}$ M. E. Rudd, Radiat. Res. 109, 1 (1987).

${ }^{11}$ M. E. Rudd, Phys. Rev. A 38, 6129 (1988).

${ }^{12}$ U. Fano and W. Lichten, Phys. Rev. Lett. 14, 627 (1965).

${ }^{13}$ M. E. Rudd, Phys. Rev. A 20, 787 (1979).
${ }^{14}$ J. B. Crooks and M. E. Rudd, Phys. Rev. A 3, 1628 (1971).

${ }^{15}$ D. K. Gibson and I. D. Reid, J. Phys. E 17, 1227 (1984); J. Phys. B 19, 3265 (1986); Radiat. Res. 112, 418 (1987); Australian Atomic Energy Commission Report No. AAEC/E659, 1987 (unpublished).

${ }^{16}$ Wen-Qin Cheng, M. E. Rudd, and Ying-Yuan Hsu, Phys. Rev. A 39, 2359 (1989).

${ }^{17}$ Yong-Ki Kim and Mitio Inokuti, Phys. Rev. A 7, 1257 (1973).

${ }^{18}$ K. Siegbahn, C. Nordling, G. Johansson, J. Hedman, P. F. Heden, K. Hamrin, U. Gelius, T. Bergmark, L. O. Werme, R. Manne, and Y. Baer, ESCA Applied to Free Molecules (North-Holland, Amsterdam, 1969).

${ }^{19}$ K. Kimura, S. Katsumata, Y. Achiba, T. Yamazaki, and S. Iwata, Handbook of HeI Photoelectron Spectra of Fundamental Organic Molecules (Halstead, New York, 1981).

20J. Macek, Phys. Rev. A 1, 235 (1970).

${ }^{21}$ J. William McGowan, Rev. Sci. Instrum. 38, 285 (1967).

${ }^{22}$ T. L. Criswell, L. H. Toburen, and M. E. Rudd, Phys. Rev. A 16, 508 (1977).

${ }^{23}$ M. E. Rudd, L. H. Toburen, and N. Stolterfoht, At. Data Nucl. Data Tables 23, 405 (1979). 